\title{
Hyperspectral Data Classification Based on EMAP and Multi- Scale Cloud Model
}

\author{
Yugui Zhao ${ }^{1}$ and Wenxing Bao ${ }^{1, *}$ \\ ${ }^{1}$ School of Computer Science and Engineering, North Minzu University, \\ Yinchuan, Ningxia 750021, P. R. China \\ *bwx71@163.com
}

\begin{abstract}
With the development of hyperspectral remote sensing classification technology, how to obtain higher classification accuracy under the condition of small samples has become a difficult and hot spot in the field of hyperspectral remote sensing technology. In recent years, Cloud model has been used in hyperspectral data analysis. In this paper, a method of hyperspectral remote sensing image classification based on EMAP (Extended MultiAttribute Profile) and cloud model is proposed. Firstly, EMAP is a kind of texture feature which is obtained by a series of attribute filters filtering hyperspectral image, and is fused with spectral feature. The fused feature is processed by LDA to reduce the dimension, and the data of dimension reduction is taken as the final classification data. Finally, according to the training sample set, the inverse cloud generator is used to generate the multi dimension cloud model for each object, and different scales are added into different cloud models to form multi-scale cloud model. Then, the membership degree of each pixel is calculated by multi-scale cloud model. Finally, we use the maximum decision method to classify each test sample. The experimental results show that the method is simple and efficient, and the classification accuracy is improved effectively.
\end{abstract}

Keywords: Linear Discriminant Analysis; Extended Multi-Attribute Profile; MultiScale Cloud model; Hyperspectral remote sensing image classification

\section{Introduction}

The hyperspectral images (HSI) use perfect sensors that extract more than a hundred of images, with more detailed information than using traditional multispectral data [1]. The contents of hyperspectral image processing include image fusion, mixed pixel decomposition, classification, target detection, physical and chemical parameters estimation and so on $[2,3,4]$. In many image processing tasks, classification is regarded as a common concern of experts and scholars in the past decades, and its goal is to assign a unique class identifier to each pixel. At present, the challenges of hyperspectral image classification include: lack of training samples, high dimensionality, uncertainty and fuzziness of data, the complementarity problem with spectral information and spatial information, as well as the complexity of the classifier.

Aiming at the lack of training samples, the uncertainty and fuzziness of the data and the complexity of the classifier: in recent years, the cloud model has been used in hyperspectral image classification because of its low demand for training samples, the uncertainty and ambiguity of data processing and high efficiency and so on. The cloud theory is applied to the classification of remote sensing images [5], which not only improves the uncertainty and fuzziness of classification, but also improves the classification accuracy. Analogously, the cloud model is applied to hyperspectral remote

Received (October 5, 2017), Review Result (December 1, 2017), Accepted (March 1, 2018)

* Corresponding Author 
sensing image classification [6] and the result show that the method is simple and the calculation is small. Its classification accuracy can be higher than the traditional classification methods.

For the high dimension problem: LDA(Linear Discriminant Analysis) [7] is a supervised linear dimension reduction algorithm. It can be used to reduce the dimension of hyperspectral data quickly. Moreover, the data points after dimension reduction can be distinguished as easily as possible. Palm vein feature is extracted through using TwoDimensional Linear Discriminant Analysis [8], which proves LDA can not only obtain the best performance in term of EER, but achieve recognition rate. The fuzzy LDA is used for face recognition [9]. The experiment proved that, the face recognition rate is greatly improved through the LDA processing. Radial basis function neural networks are used to classify the plastic materials into several categories based on the obtained spectra reduced by principal component analysis and linear discriminant analysis techniques [10], and better classification results are obtained.

According to the complementarity problem with spectral information and spatial information: The spatial structure features from the image can be extracted by morphological operation to form the feature space being used to describe the image structure attribute information: EMAP. It is an effective way of spectral and spatial classification. The validity of the morphological attribute profile in the extraction of spatial information with two high-resolution hyperspectral images is proved [11]. The classification for the remote sensing image based on the sparse representation of the morphological attributes is recommended [12]. A spectral and spatial classification framework for hyperspectral image is proposed [13], using morphological attributes to obtain spatial information, using two kinds of supervised methods to extract the feature of spatial information, and using Random Forest to classify hyperspectral image. A method to automatically generate the standard deviation of the morphological attributes profiles based on sample statistics is established [14]. The above all studies have achieved good results.

In this paper, a new algorithm for hyperspectral remote sensing image classification based on EMAP and multi-scale cloud is proposed. The combination of spectral features and morphological properties of EMAP, which makes full use of the spectral information and spatial information of hyperspectral data, is then projected onto the optimal vector space by LDA. The experimental results show that the classification accuracy of hyperspectral data is improved significantly.

\section{Hyperspectral Data Classification based on EMAP and Multi-Scale Cloud Model}

\subsection{EMAP}

The Extended Multi-AttributeProfile algorithm is a feature extraction algorithm based on the morphological attributes filtering. The basic idea is to use a series of different morphological filters to filter the image to extract the structure information of the image. Then, the spatial structure of the image is obtained by integrating the filtering results of different attributes. The property $\mathrm{Ci}$ of the connected components of the gray image $\mathrm{f}$ is evaluated by the attribute filtering operation through a given reference threshold $\lambda$. For example, the connected component of the image is $\mathrm{Ci}$. If the property value $\mathrm{AP}(\mathrm{Ci})>\lambda$, the region remains unchanged. Instead, the $\mathrm{Ci}$ will be assigned to a neighboring value with merging $\mathrm{Ci}$ into the surrounding sub blocks. If the gray value of the merged region is low, the process becomes the thinning transformation of the image, instead of becoming thickening transformation, $\mathrm{AP}$ is defined as:

$$
\mathbf{A P}(f):=\left\{\varphi_{n}(f), \cdots, \varphi_{1}(f), f, \gamma_{1}(f), \cdots, \gamma_{n}(f)\right\}
$$




$$
\mathbf{E A P}=\left\{\mathbf{A P}_{\mathbf{1}}, \mathbf{A} \mathbf{P}_{2}, \cdots, \mathbf{A} \mathbf{P}_{\mathbf{i}}\right\}
$$

Formula: APi $(i=1, \ldots, n)$ represents the property filtering of component $i$, where $n$ represents the number of principal components retained. $\varphi \mathrm{i}$ and $\gamma \mathrm{i}$ represent thickening operation and thinning operation respectively. Figure 1 shows the structure of EAP.

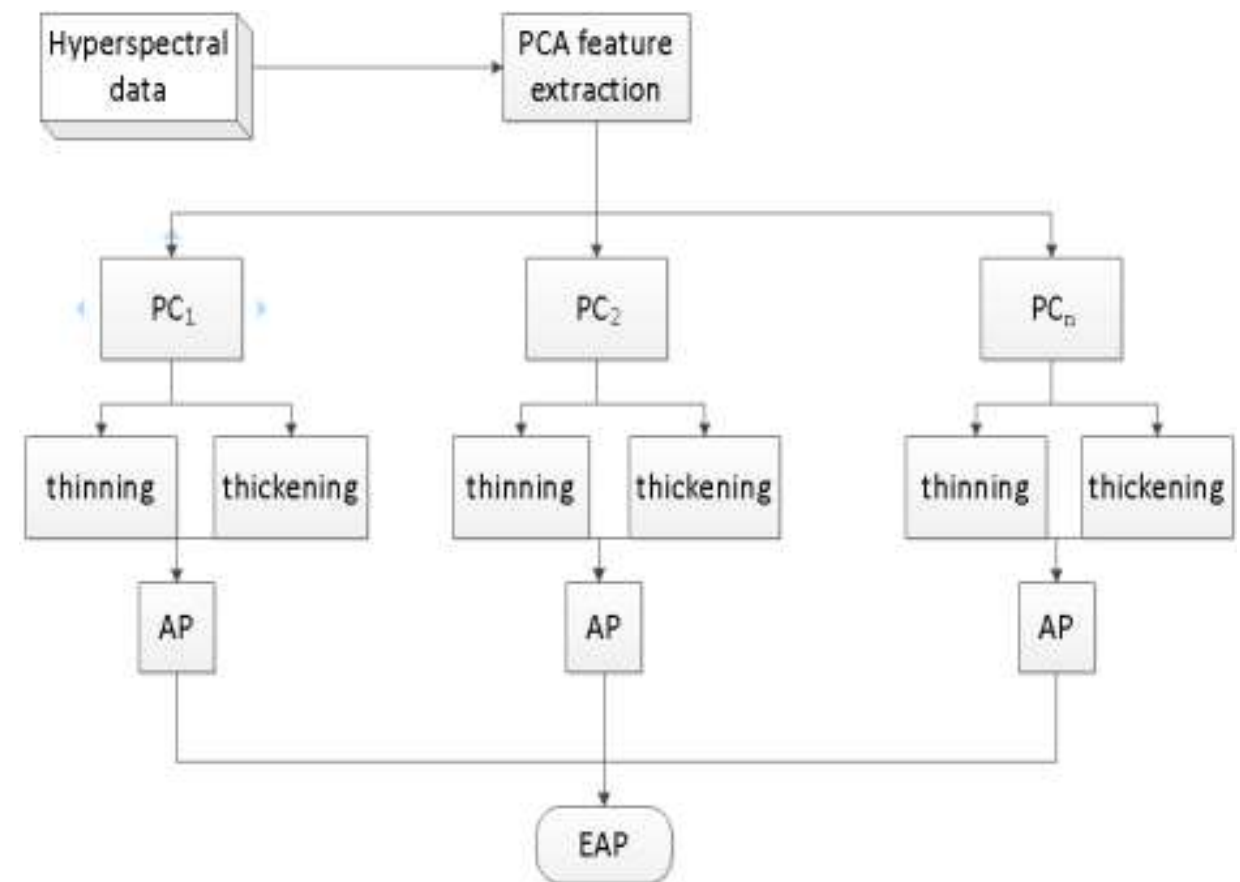

Figure 1. Extended Attribute Profile Generation

Five principal components are extracted by PCA (total information is more than 99\%) to build extended multi-attribute profiles. In the paper, four attribution profiles (Area criterion, Pixel standard deviation criterion, Moment of inertia, Shape criterion) are extracted to form EMAP. The specific settings for these four parameters are as follows: (1) Area criterion: $\lambda \mathrm{a}=[1005001000$ 5000]; (2) Pixel standard deviation criterion: $\lambda s=[20$

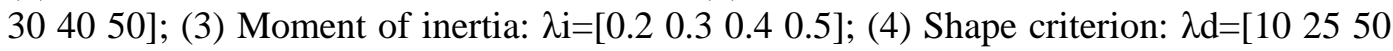
100].

$$
\mathbf{E A M P}=\left[\operatorname{EAP}_{\mathrm{a}}, \text { EAP }_{\mathrm{s}}, \mathbf{E A P}_{\mathrm{i}}, \mathbf{E A P}_{\mathrm{d}}\right]
$$

\subsection{Cloud Concept and Algorithm Description}

2.2.1. Cloud Concept: Cloud is an uncertain transformation model between a qualitative concept and its quantitative representation expressed by linguistic value. It mainly reflects two uncertainty of the concept of the universe or human cognition: fuzziness (real and imaginary) and randomness (probability of occurrence). 


\subsubsection{Multi-Scale Cloud Model}

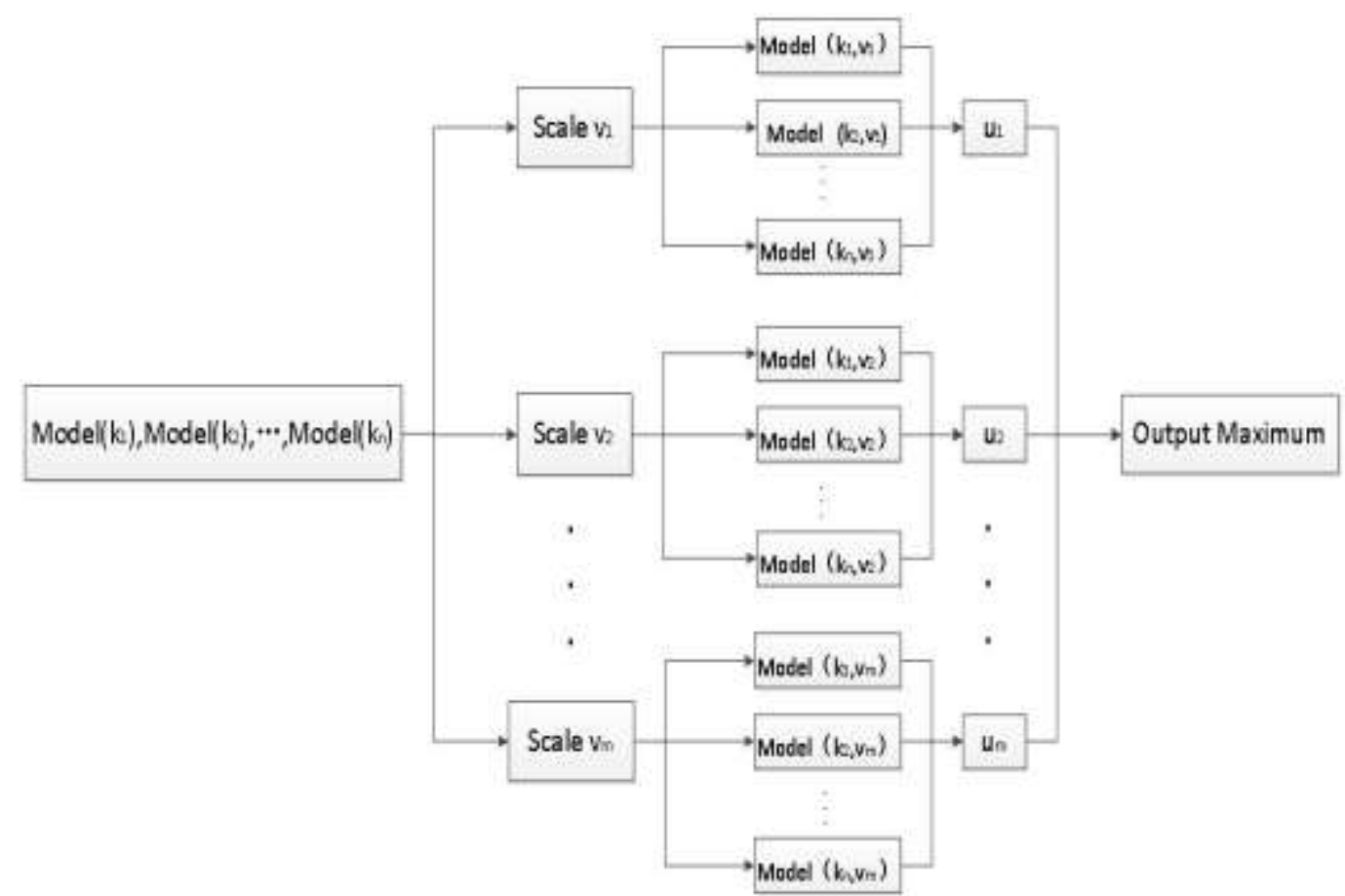

Figure 2. Multi-Scale Cloud Model

In Figure 2, $v_{m} k_{n}$ denote the first $\mathrm{m}$ scale and the first $\mathrm{n}$ cloud model respectively. $\operatorname{Model}\left(k_{n}, v_{m}\right)$ denotes the first $n$ model with $v_{m}$ scale. $u_{m}$ represents the maximum membership degree in $n$ models under scale $v_{m}$.

\subsubsection{Algorithm Description: Step 1: feature extraction and feature fusion}

Step 1.1: Hyperspectral data: $\mathbf{H}=\operatorname{matrix}(n, m) ; \% n$ and $m$ are sample dimension and sample number respectively.

Step 1.2: For $i=1, \ldots, n$ in $\mathbf{H}$ do

First, dimension reduction based on PCA; Sencond, obtaining EMAP (spatial feature) extracted by morphological filtering operators;

End for

Step 1.3: Fusing spectral features and spatial features; The fused features reduced by LDA being as final classification data.

Step 2: The construction of Cloud model

For $k=1, \ldots, N$ do $\% N$ represents the number of classes.

Input: Training sample number of class $k: N_{k}$, training sample matrix $\mathrm{X}_{\mathrm{k}}=\left[x_{k 1}, x_{k 2}, \ldots, x_{k N k}\right]$;

Step 2.1: Sample mean: $\overline{X_{k}}=\frac{1}{N_{k}} \sum_{i=1}^{N_{k}} x_{k i}$

Step 2.2: Absolute center distance of first order sample: $d_{k}=\frac{1}{N_{k}} \sum_{i=1}^{N}\left|x_{k i}-\overline{X_{k}}\right|$

Step 2.3: Sample variance: $S_{k}=\frac{1}{N_{k}-1} \sum_{i=1}^{N_{k}}\left(x_{k i}-\overline{X_{k}}\right)^{2}$ 
Step 2.4: Expectation: $E_{k x}=\overline{X_{k}}$

Step 2.5: Entropy: $E_{k n}=\sqrt{\frac{p i}{2}} \times \frac{1}{N_{k}} d_{k}$

Step 2.6: Hyper entropy: $H_{k e}=\sqrt{S_{k}-E_{k n}^{2}}$

Output: The Three digital features of the $k$ Cloud model: Expectation $E_{k x}$, Entropy $E_{k n}$, Hyper entropy $H_{k e}$.

End for.

Step 3: Construction of Multi-Scale Cloud model and calculation of membership Input: Testing sample to be classified $x, u_{k}, u_{0}$.

Step 3.1: The following formula $E=\operatorname{normrnd}\left(E_{k n}, H_{k e}\right)$ can generate normal random numbers. $\% E_{k n}$ and $H_{k e}$ denote expectation value and standard deviation respectively. the membership degree of class $k$ that a sample belongs to: $u_{k v}=\exp \left[-\frac{v\left(x-E_{k x}\right)^{2}}{10 E^{2}}\right]$. In the formula, scale $v \in(0,1)$, The step length of $v$ is 0.05 .

Step 3.2: Pseudo codes for constructing Multi-Scale Cloud model

$$
\begin{aligned}
& \text { For } k=1, \ldots, N \text { do } \\
& \text { For } v=0.1, \ldots, 1 \text { do } \\
& u_{k v}=\exp \left[-\frac{v\left(x-E_{k x}\right)^{2}}{10 E^{2}}\right] \\
& u_{k}=u_{k v} \text { If } u_{k v}>u_{k} \\
& \text { End for } \\
& u_{0}=u_{k} \text { if } u_{k}>u_{0}
\end{aligned}
$$

End for

Output $u_{0}$

In the upper form, the $v$ is $0.1,0.15,0.20$ and so on when the step size is 0.05 . The following Figure 3 and Figure 4 are the effect of scale number on the total classification accuracy under different training samples on two hyperspectral data.

From the Figures 3 and 4, it can be seen that the four curves in the two figures increase first and then decrease with the increasement of the scale number. In addition, it can be seen clearly from the two figures that when the number of scales reaches 10 or 11 , the over accuracy of different training samples is the nearly highest. What's more, when the number of scales is 11 , the accuracy of the 5 training samples is the highest. Therefore, in this paper, five training samples are taken in each class, and the scale number is set to 11 . 


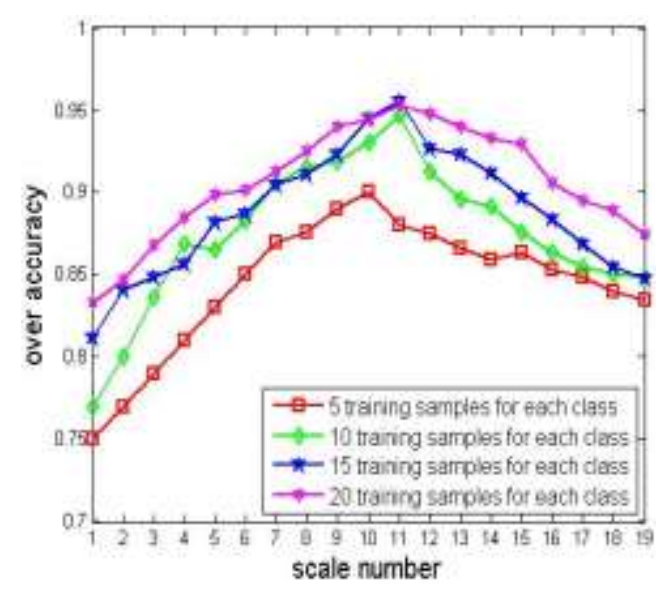

Figure 3. AVIRIS Data Experiment

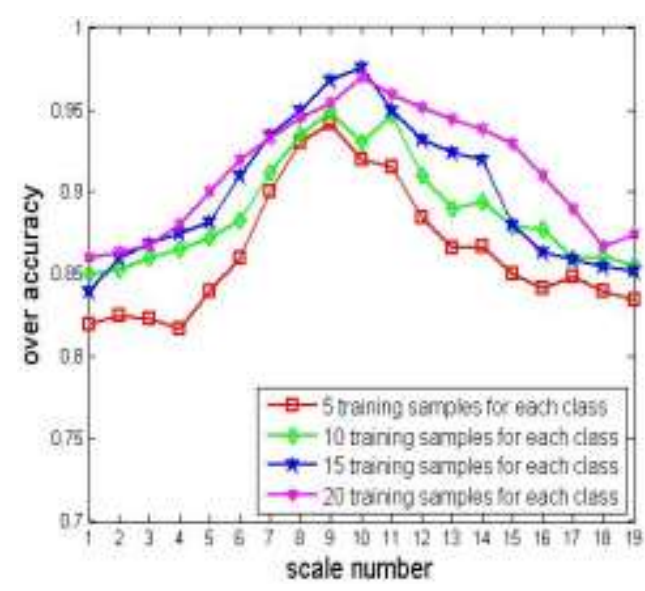

Figure 4. ROSIS Data Experiment

\subsection{Classification Algorithm Model}

The classification algorithm proposed in this paper consists of 4 key links: construction of spatial feature vector (EMAP), fusion of spectral feature and spatial feature, dimension reduction of fusion data by LDA, prediction classification of Multi-Scale Cloud model generated by training samples. The specific technical process is shown in Figure 5.

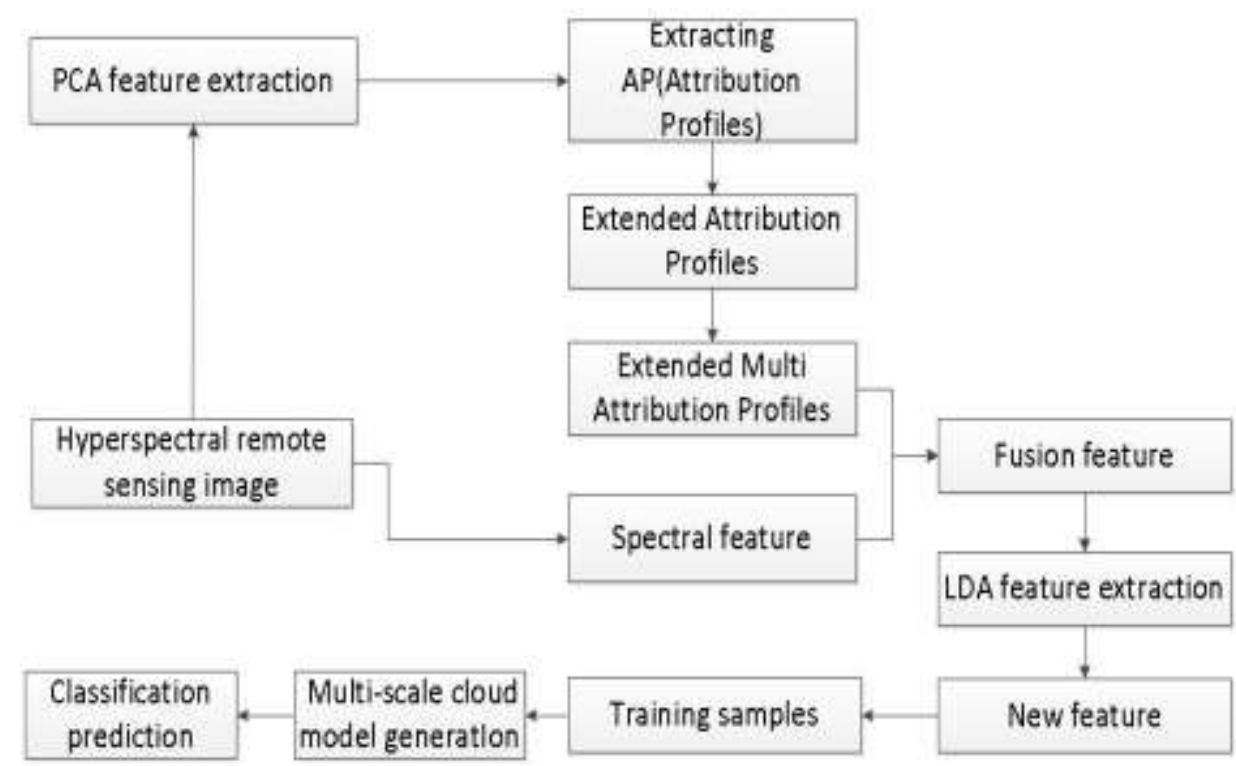

Figure 5. Classification Algorithm Model

\section{Experiment and Discussion}

\subsection{Evaluating Indicator}

In the following formula, $\left(x_{11}, x_{22}, \ldots, x_{n n}\right)$ is the number of samples that are assigned to the correct class with $n$ representing the total number of categories. $x_{i j}$ is the number of classified samples of class $i$ and reference data type $j$ in classified data. $x_{i+}=\sum_{j=1}^{n} x_{i j}$ obtained by classification is the sum of the class $i . x_{+j}=\sum_{i=1}^{n} x_{i j}$ is the sum of the class $j$ of reference data. $N$ is the total number of samples. In this paper, four evaluation indexes can be used to evaluate the classification results: 
User Accuracy: the ratio of the number of samples that are correctly classified into class $i$ and the total number of samples assigned to class $i$

$$
U A=\frac{x_{i i}}{x_{i+}}
$$

Over Accuracy: equaling to the total number of pixels divided by the number of pixels that are correctly classified.

$$
O A=\sum_{k=1}^{n} \frac{x_{k k}}{N}
$$

Average Accuracy: the ratio of the sum of the user accuracy of all classes and the total class numbers

$$
A A=\underline{\sum_{i=1}^{n} \frac{x_{i i}}{x_{i+}}}
$$

Kappa coefficient: in the statistical sense, the classification results are better than the random classification results, which can be used to measure whether the error matrix of the two classifiers have significant differences.

$$
K=\frac{N \sum_{i=1}^{n} x_{i i}-\sum_{i=1}^{n}\left(x_{i+} x_{+i}\right)}{N^{2}-\sum_{i=1}^{n}\left(x_{i+} x_{+i}\right)}
$$

Table 1. List of Abbreviations Used in this Paper

\begin{tabular}{l|l}
\hline SVM & Support Vector Machine \\
EMAP & Extended Multi-Attribute Profile(spatial feature) \\
SPE & Spectral feature \\
Cloud & Cloud model in this paper \\
MSC & Multi-Scale Cloud model being proposed in the paper \\
SE & SVM with EMAP \\
SSE & SVM with SPE and EMAP \\
CE & Cloud with EMAP \\
CSE & Cloud with SPE and EMAP \\
MSCE & MSC with EMAP \\
MSCSE & MSC with SPE and EMAP \\
\hline
\end{tabular}

\subsection{First Experiment}

3.2.1. AVIRIS Indian Pines Data: Indian Pines AVIRIS hyperspectral remote sensing image in June 1992 is applied in our experiment. This image is composed of $145^{*} 145$ pixels, and spatial resolution is about $20 \mathrm{~m} /$ pixel. 220 spectral bands are included in the range of 0.4 to $2.5 \mathrm{um}$. The data set include sixteen types of objects. Figure 6 shows the hyperspectral data for Indian Pines. 


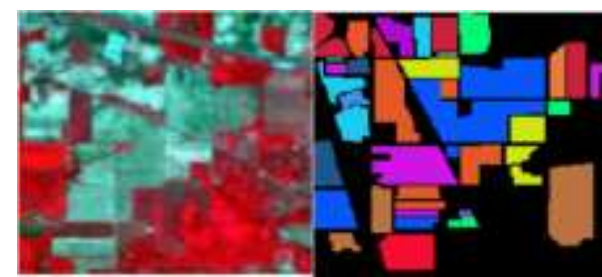

(a) False Color Image (b) validation Data

\section{Figure.6 AVIRIS Indian Pines Data}

3.2.2. AVIRIS Analysis: Figure 7 is the classification results of the six cases when 10 training samples for each object is selected from the AVIRIS data. As can be seen, several conclusions can be obtained from Figure 7. First and foremost, the proposed MSCSE algorithm clearly obtained the best results in all cases. This is due to the fact that the proposed approach not only takes advantage of both spatial and spectral information, but combine the multi-scale factor. as opposed to the other tested approaches (SE, CE and MSCE) which use spatial information alone. In addition, the proposed MSCSE provides results which are comparable to those obtained by other spectral-spatial methods(SSE and CSE). From Table 2, it can be observed that all spectral-spatial based algorithms (SSE, CSE and MSCSE) exhibit good performance, indicating the importance of including spatial information in the analysis. Table 2 shows the OA, AA, $k$ statistic, and individual class accuracies obtained using 160 training samples, 10 samples of each class in the ground-truth image. It is worth noting that, in this challenging classification scenario, the MSCSE algorithm achieved better performance than the other spectral-spatial algorithm(CSE and MSCSE). Overall, the experiments suggest that the proposed MSCSE is competitive with some of the best available spectral-spatial methods for hyperspectral image classification. Based on Figure 7 and table 1, the classification method taking into account of fuzziness and randomness of the concept and multi-scale factors simultaneously has a great advantage.

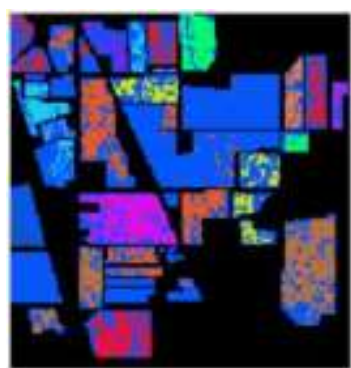

(a) SE $(63.30 \%$ c)

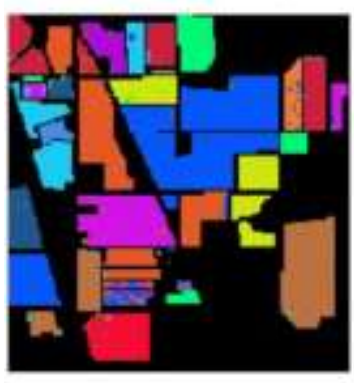

(d) CSE (95.50\% \%)

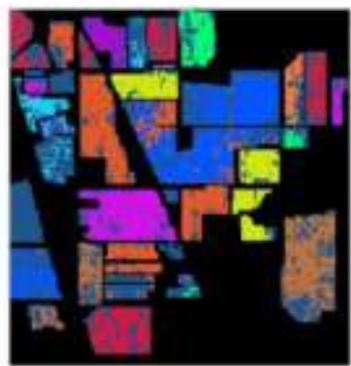

(b) SSE $(67.12 \%)$

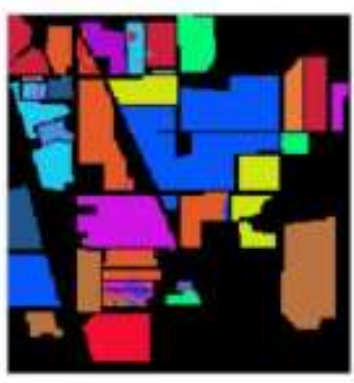

(e) MSCE (96.49\%;

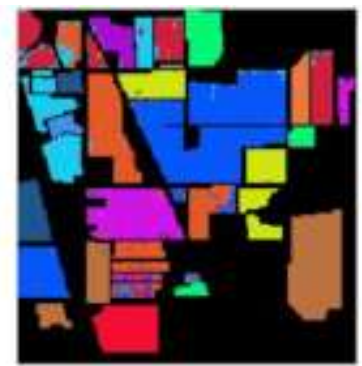

(c) $C E(93.37 \%$ )

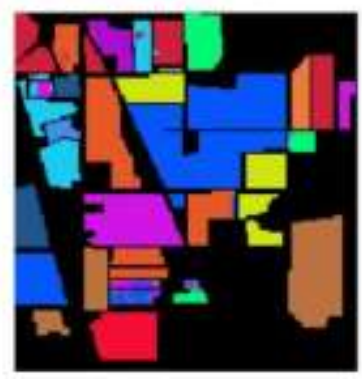

(f) MSCSE $\left(97.61^{\circ} \%\right.$

Figure 7. Classification Results of Different Classification Methods of AVIRIS Data under the Condition of 10 Training Samples per Class 
Table 2. The Accuracy of Different Classification Methods of Indiana in the Selection of 10 Training Samples per Class

\begin{tabular}{|c|c|c|c|c|c|c|c|c|c|}
\hline Number & Class & Training samples & Testing samples & SE & SSE & $\mathrm{CE}$ & CSE & MSCE & MSCSE \\
\hline 1 & Alfalfa & 10 & 44 & 31.82 & 1364 & 100.00 & 86.36 & 88.14 & 86.75 \\
\hline 2 & Corn-no till & 10 & 1424 & 56.17 & 6832 & 84.20 & 93.05 & 91.68 & 92.58 \\
\hline 3 & Corn-min till & 10 & 824 & 48.59 & 5565 & 89.81 & 91.99 & 98.24 & 99.29 \\
\hline 4 & Corn & 10 & 224 & 26.64 & 5935 & 93.75 & 96.43 & 95.93 & 97.67 \\
\hline 5 & Grass / pasture & 10 & 487 & 64.77 & 60.80 & 96.51 & 100.00 & 96.45 & 97.42 \\
\hline 6 & Grass / tree & 10 & 737 & 40.22 & 65.06 & 98.24 & 97.15 & 99.99 & 100.00 \\
\hline 7 & Grass / pasture-mowed & 10 & 16 & 53.33 & 18.75 & 87.50 & 93.75 & 95.00 & 95.63 \\
\hline 8 & Hay-windrowed & 10 & 479 & 62.18 & 77.40 & 99.37 & 97.29 & 99.69 & 99.77 \\
\hline 9 & Oats & 10 & 10 & 20.00 & 20.00 & 100.00 & 100.00 & 100.00 & 100.00 \\
\hline 10 & Soybeans-no till & 10 & 958 & 60.74 & 64.03 & 82.99 & 88.73 & 90.37 & 94.62 \\
\hline 11 & Soybeans-min till & 10 & 2458 & 97.95 & 67.93 & 94.79 & 97.84 & 97.48 & 98.89 \\
\hline 12 & Soybeans-clean till & 10 & 604 & 38.58 & 69.70 & 98.51 & 96.69 & 95.07 & 96.19 \\
\hline 13 & Wheat & 10 & 202 & 57.81 & 60.94 & 99.50 & 89.11 & 99.54 & 99.54 \\
\hline 14 & Woods & 10 & 1284 & 63.26 & 68.29 & 99.14 & 97.90 & 99.93 & 99.95 \\
\hline 15 & Bldg-grass-tree-drives & 10 & 370 & 17.27 & 100.00 & 99.19 & 95.95 & 99.26 & 99.42 \\
\hline 16 & Stone-steel towers & 10 & 85 & 44.00 & 65.33 & 95.29 & 100.00 & 98.13 & 98.87 \\
\hline \multicolumn{4}{|c|}{$O A / \%$} & 63.30 & 67.12 & 93.37 & 95.50 & 96.49 & 97.61 \\
\hline \multicolumn{4}{|c|}{$A A / \%$} & 48.96 & 58.45 & 94.92 & 95.14 & 96.55 & 97.31 \\
\hline \multicolumn{4}{|c|}{ Kappa coefficient } & 55.13 & 60.32 & 92.44 & 94.86 & 95.99 & 97.27 \\
\hline
\end{tabular}

\subsection{Second Experiment}

3.3.1. ROSIS University of Pavia Data: The Italy University of Pavia data collected by ROSIS spectral imager is applied in our experiment. The spatial resolution was $1.3 \mathrm{~m}$. The image size is $610 \times 340$ pixels, and the wavelength range of $0.43-0.86 u m$ is about 115 bands. After removal of the noise band, 103 bands are usually used for classification. 9 typical objects in the sample of 42776 are contained in the image. Figure 8 shows the data of University of Pavia in Italy.

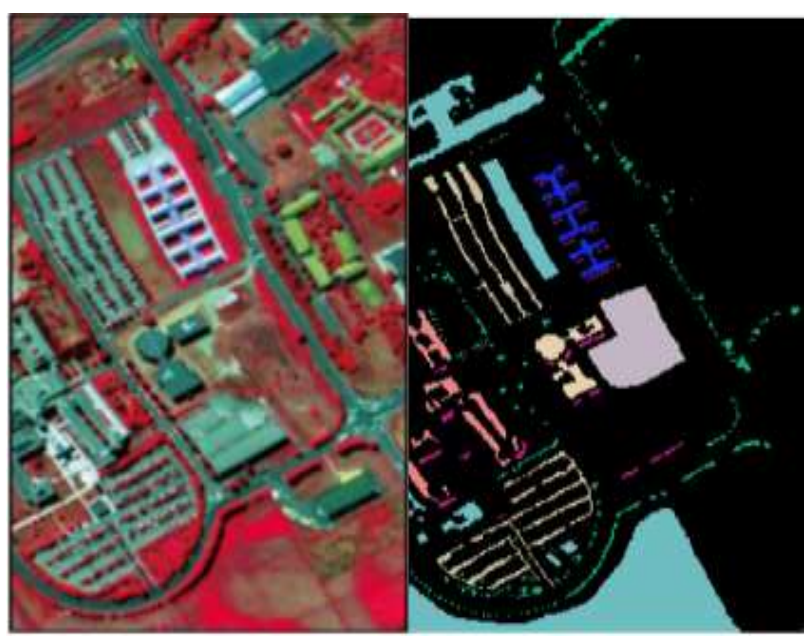

(a) False Color Image (b) Surface Validation Data

Figure 8. ROSIS University Pavia Data 
3.3.2. ROSIS Analysis: In this experiment, we conduct an evaluation of the proposed MSCSE algorithm with regard to other better hyperspectral image classification methods. Table 3 shows the OA, AA, $K$, and individual class accuracies obtained in our algorithm comparison, which includes well known spectral-based classification methods such as SVM and Cloud model. It can be seen from Table 3 that our classification method is better than other classification methods mentioned in this paper. This is because proposed MSCSE contain uncertainty and fuzziness of things, which is of great help to improve the ability of differentiating intra-class. More importantly, proposed MSCSE include multiscale factors, which can simulate complex distribution of hyperspectral data more effectively, improving classification accuracies.

In order to further illustrate the good performance of the proposed approach, Figure 9 lists the classification results of the six cases when the 10 training samples per class is selected from the ROSIS data. Figure 9 shows the six obtained classification maps corresponding to Table 3. It's not difficult to see that proposed MSCSE still has low error rate and better classification effect than other approches mentioned in this paper. For example, the classification accuracy of proposed MSCSE is $7.22 \%$ and 1.09\% higher than that of SSE and CSE, respectively. Besides, the more important discovery is that the proposed method has higher classification performance than consistent with other methods in this paper, whether it contains only spatial information or spectral-spatial information classification. The reason is that it not only contains uncertainty and fuzziness, but also incorporates multi-scale factors.

Table 3. Accuracy of Different Classification Methods of Italy University of Pavia at the Time of Selecting 10 Training Samples per Class

\begin{tabular}{|c|c|c|c|c|c|c|c|c|c|}
\hline Number & Class & Training samples & Testing samples & SE & SSE & $\mathrm{CE}$ & CSE & MSCE & MSCSE \\
\hline 1 & Bitumen & 10 & 6621 & 81.52 & 90.94 & 99.21 & 98.84 & 99.48 & 99.61 \\
\hline 2 & Meadows & 10 & 18639 & 96.08 & 93.17 & 98.32 & 99.34 & 99.87 & 99.91 \\
\hline 3 & Gravel & 10 & 2089 & 75.77 & 90.86 & 99.33 & 99.66 & 99.68 & 99.51 \\
\hline 4 & Trees & 10 & 3054 & 79.93 & 99.97 & 97.39 & 98.63 & 98.92 & 99.60 \\
\hline 5 & Metal sheets & 10 & 1335 & 68.10 & 74.80 & 96.65 & 97.03 & 99.85 & 99.86 \\
\hline 6 & Bare soil & 10 & 5019 & 79.94 & 96.34 & 97.57 & 97.81 & 99.98 & 99.99 \\
\hline 7 & Asphalt & 10 & 1320 & 89.62 & 93.61 & 87.59 & 92.26 & 99.92 & 99.92 \\
\hline 8 & Bricks & 10 & 3672 & 95.30 & 95.22 & 95.78 & 99.08 & 99.63 & 99.64 \\
\hline \multirow[t]{3}{*}{9} & Shadows & 10 & 937 & 100.00 & 65.82 & 98.94 & 98.20 & 99.39 & 99.48 \\
\hline & $O A / \%$ & & & 88.71 & 92.58 & 97.76 & 98.71 & 99.71 & 99.80 \\
\hline & $A A / \%$ & & & 85.14 & 88.97 & 96.75 & 97.87 & 99.64 & 99.73 \\
\hline \multicolumn{2}{|c|}{ Kappa coefficient } & & & 85.33 & 90.31 & 97.04 & 98.29 & 99.62 & 99.73 \\
\hline
\end{tabular}




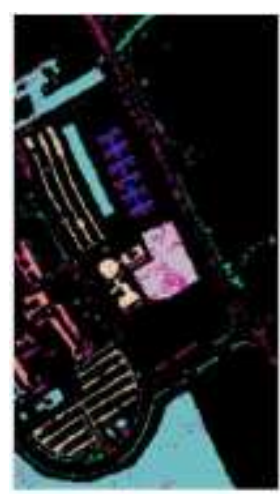

(a) $S E(88.71 \%)$

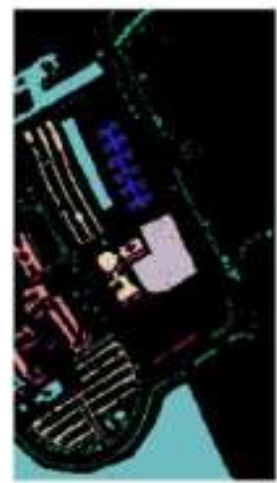

(d) CSE (98. $71^{\circ} \%$

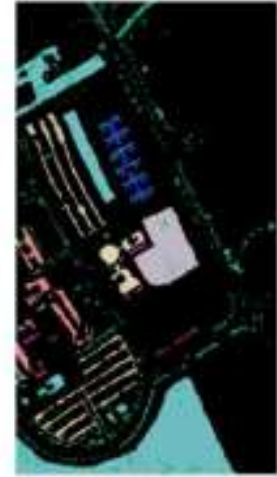

(b)SSE( $92.58 \%$ )

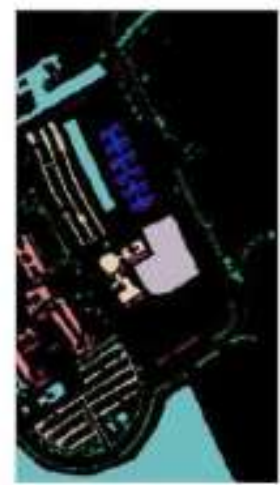

(e) MSCE $(99.71 \%)$

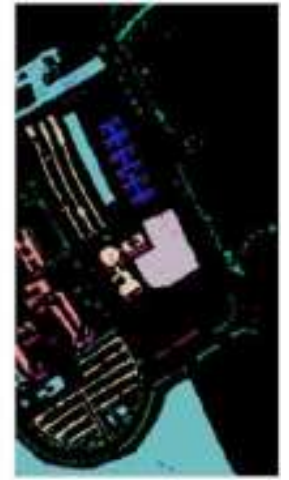

(c) $\mathrm{CE}\left(97.76^{\circ} \%\right)$

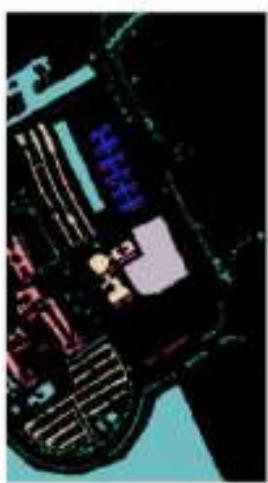

(f) MSCSE (99.80\%。)

\section{Figure 9. Classification Results of Different Classification Methods of ROSIS Data under the Condition of 10 Training Samples per Class}

In order to illustrate the advantages of proposed classification method under different training samples, Figure 10 and Figure 11 are the comparison of the over accuracy of different classification methods of AVIRIS data under different samples numbers selected from per class. Figure 11 is the comparison of the over accuracy of different classification methods of ROSIS data under different samples selected from per class. With the increasement of samples number, it can be drawn from the two figures: (1) the over accuracy of SE and SSE is on the rise, and very volatile and unstable. In addition, under the condition of the training sample fixed, the latter is slightly higher than the former. This indicates that spectral information is very important in hyperspectral image classification. (2) The results of CE, CSE, MSCE and proposed MSCSE are relatively stable, and the total accuracy is far greater than that of SE and SSE. Proposed MSCSE has the highest precision in all classification approaches. At the same time, it is not difficult to see that the classification accuracy of the proposed MSCSE classification method has reached the highest level. This shows that proposed MSCSE has great advantages in small sample numbers. 


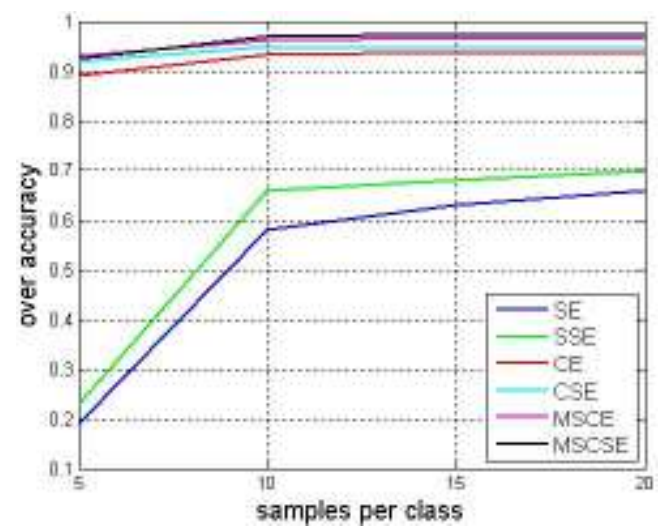

Figure 10. Comparison of the Overall Accuracy of Different Classification Methods of AVIRIS Data Under Different Sample Conditions

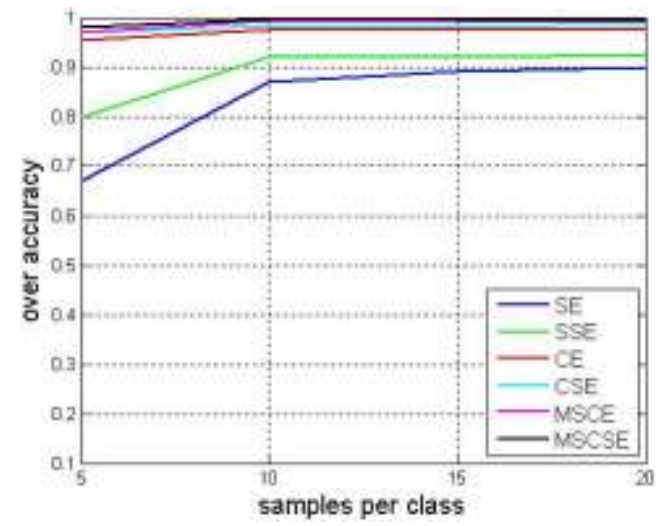

Figure 11. Comparison of the Overall Accuracy of Different Classification Methods of ROSIS Data Under Different Sample Conditions

\section{Conclusions and Prospect}

In this paper, a new hyperspectral image classification method is proposed. Firstly, original spectral features and morphological features EMAP are combined. Secondly, the combined features are projected to the best vector space by LDA, and the data in the new vector space are classified by the multi-scale cloud model. The experiments of two hyperspectral images classification show that the proposed algorithm can achieve better classification results. In future work, we will explore other representative features and design a better classification model to further improve the classification accuracy.

\section{Acknowledgment}

This work is supported by National Natural Science foundation of China (Project No. 61461003).

\section{References}

[1] H. Nhaila, E. Sarhrouni and A. Hammouch, "A Survey on Fundamental Concepts and Practical Challenges of Hyperspectral Images", 2014 Second World Conference on Complex Systems (WCCS), Agadir, Morocco, (2014) November 12-14.

[2] J. M. Bioucas-Dias, A. Plaza, G. Camps-Valls and P. Scheunders, "Hyperspectral Remote Sensing Data Analysis and Future Challenges", IEEE Geoscience and Remote Sensing Magazine, vol. 1, no. 2, (2013), pp. 6-36. 
[3] A. Plaza, J. A. Benediktsson and J. W. Boardman, "Recent Advances in Techniques for Hyperspectral Image Processing", Remote Sensing of Environment, vol. 113, no. 9, (2009), pp. S110-S122.

[4] Z. Zhou, "Progress in Hyperspectral Remote Sensing for Petroleum Prospecting", Remote Sensing Technology and Application, vol. 29, no. 2, (2014), pp. 352-361.

[5] L. Lin and G. Ding, "A multiple classification method based on the cloud model", Neural Network World, vol. 20, no. 5, (2010), pp. 651-666.

[6] Z. Wu, Y. Li, A. Plaza, J. Li and F. Xiao, "Parallel and Distributed Dimensionality Reduction of Hyperspectral Data on Cloud Computing Architectures", IEEE Journal of Selected Topics in Applied Earth Observations and Remote Sensing, vol. 9, no.6, (2016), pp. 2270-2278.

[7] X. Zhang, D. Chu and R. C. E. Tan, "Sparse Uncorrelated Linear Discriminant Analysis for Undersampled Problems", IEEE Transactions on Neural Networks and Learning Systems, vol. 27, no. 7, (2016), pp. 1469-1485.

[8] F. Rizki, T. A. B. Wirayuda and K. N. Ramadhani, "Identity Recognition based on Palm Vein Feature Using Two-Dimensional Linear Discriminant Analysis", International Conference on Information Technology, Information Systems and Electrical Engineering (ICITISEE), Yogyakarta, Indonesia, (2016) August 23-24.

[9] G. Y. Zhang, "Face Recognition based on Fuzzy Linear Discriminant Analysis", IERI Procedia, vol. 2, (2012), pp. 873-879.

[10] S. B. Roh, S. K. Oh, E. K. Park and W. Z. Choi, "Design of Radial Basis Function Neural Networks with Principal Component Analysis and Linear Discriminant Analysis for Black Plastic Identification", 2016 Joint 8th International Conference on Soft Computing and Intelligent Systems (SCIS) and 17th International Symposium on Advanced Intelligent Systems (ISIS), Sapporo, Japan, (2016) August 25-28.

[11] M. D. Mura, J. A. Benediktsson, B. Waske and L. Bruzzone, "Morphological Attribute Profiles for the Analysis of very High Resolution Images", IEEE Transactions on Geoscience and Remote Sensing, vol. 48, no. 10, (2012), pp. 3747-3762.

[12] B. Song, J. Li, M. D. Mura and J. Chanussot, "Remotely Sensed Image Classification Using Sparse Representations of Morphological Attribute Profiles", IEEE Transactions on Geoscience and Remote Sensing, vol.52, no.8, (2014), pp. 5122-5136.

[13] P. Ghamisi, J. A. Benediktsson, G. Cavallaro and A. Plaza, "Automatic Framework for Spectral-Spatial Classification based on Supervised Feature Extraction and Morphological Attribute Profiles", IEEE Journal of Selected Topics in Applied Earth Observations and Remote Sensing, vol. 7, no. 6, (2014), pp. 2147-2160.

[14] P. R. Marpu, M. Pedergnana, M. D. Mura, J. A. Benediktsson and L. Bruzzone, "Automatic Generation of Standard Deviation Attribute Profiles for Spectral-Spatial Classification of Remote Sensing Data", IEEE Geoscience and Remote Sensing Letters, 2010, 10(2), 293-297. IEEE transactions on Geoscience and remote sensing, vol.48, no.1, (2013), pp. 186-197.

\section{Authors}

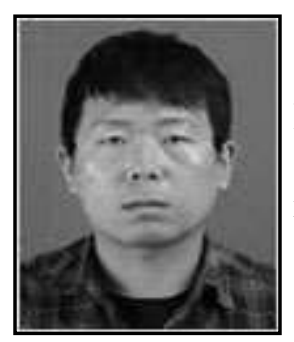

Yugui Zhao, received his B.S. degree in school of educational science and technology from Datong University, China, in 2015. Now he is working for his M.E. degree in School of Computer Science and Engineering of North Minzu University, China. His research interests concentrate on digital image processing.

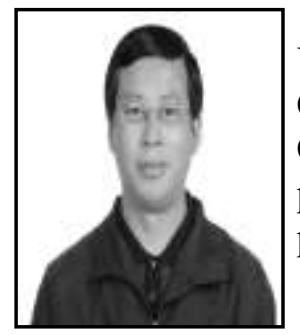

Wenxing Bao, received his Ph.D degree from Xi'An Jiaotong University, China, in 2006. He is a full professor in the School of Computer Science and Engineering of North Minzu University, China, His research interests include remote sensing image processing, AI, computer vision, and computer graphics. He has published about 50 papers in international/national journals. 
International Journal of Signal Processing, Image Processing and Pattern Recognition Vol. 11, No. 2 (2018) 